\title{
Towards a mobile serious game environment for children self- learning
}

\author{
Charles Gouin-Vallerand \\ Science and Technology \\ Télé-Université du Québec \\ Montreal, QC, Canada \\ cgouinva@teluq.ca
}

\author{
Susan Möller Ferreira \\ LICEF Research Center \\ Télé-Université du Québec \\ Montreal, QC, Canada \\ susanferreira@gmail.com
}

\author{
Richard Hotte \\ Science and Technology \\ Télé-Université du Québec \\ Montreal, QC, Canada \\ rhotte@ teluq.ca
}

\begin{abstract}
Even with all the efforts worldwide achieving universal primary education is still a crucial challenge. Aiming a contribute to solve this problem, we designed a serious game to provide education for children, as a supplement to daily school learning and/or in lieu of availability of regular schooling. A mobile serious game, introducing concepts of Mathematics and English, was developed and evaluated with children. The results showed the effectiveness of the serious game into motivating children to take part in the educational activity and suggested an improvement in their knowledge. Moreover, it highlighted aspects in the game design, pedagogical pattern, game instruction and tablet interaction that could be improved. These results have important implications for future research in this field, showing positive outcomes from the design approach and providing recommendations for further research in this field.
\end{abstract}

\section{CCS CONCEPTS}

- CCS $\rightarrow$ Human-centered computing $\rightarrow$ Collaborative and social computing $\rightarrow$ Collaborative and social computing devices

\section{KEYWORDS}

Serious games; children education; mobile learning; childrentablet interaction.

\section{INTRODUCTION}

Even with all the advances in education and technology access, there are currently around the globe 58 million children, aged from 6 to 11, not attending school [24]. Understanding the importance of this problem, previous researchers have identified

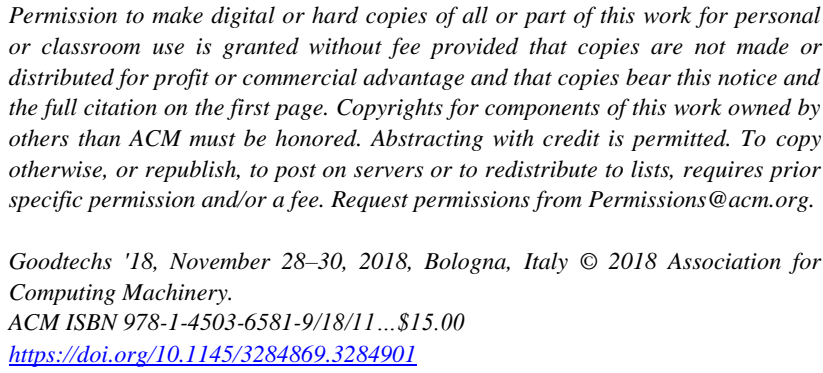

various reasons for it. In relation to Bangladesh, factors as economic, social, family, geographic conditions, racial discrimination and gender disparities are mentioned [5]. The works of Kim found similar situations in relation to the South American indigenous, with seasonal or migrant workers [14]. Analogous results were identified regarding the failure of access of young Kenyan children to primary education, caused by poverty, inequality in gender, and regional disparities [2].

In spite of the significant technology penetration increase in developing countries in the last decade, scholars called attention to the need of further work to understand how these technologies could be applied to actually impacts human development [10]. On the one hand, in the ambit of educational technology, previous researchers had shown a growing interest in serious games (e.g.: [5] [11]). In the other hand, there is not strong solution in this area that focus on providing children's education in lieu of availability of regular schooling.

In this research, we aim to contribute to this field by designing a serious game prototype that focus in children self-learning. In this paper we focus on Grade 1, introducing the first lessons to learn Mathematics and English. We aim to apply the results of this study in further research, aiming to adapt the contents of math and English subjects, offered from Kindergarten to Grade 12, in the school Curriculum onto an application embedded within a tablet PC. The application would be applied in both learning scenarios, as a supplement to daily school learning or in lieu of availability of regular schooling. This raises the challenge to offer a high level of motivation and user-friendliness for a student to effectively educate him/herself.

\section{LITTERATURE REVIEW}

\subsection{Children Game Based Learning}

The value of learning through gaming has been recognized in the children's education field for more than a decade. Previous studies showed that game-based learning is seen as an option to address students learning and motivation requirements [20] and that games implementing pedagogical design can strengthen and support children's cognitive abilities [8].. In the context of board and card games, serious games were defined as games that have 
an explicit and carefully thought-out educational purpose, and are not intended to be played primarily for amusement [1].

Previous research highlighted that to balance between fun and educational measures is a key challenge in serious game design [6] [15] Klopfer et al. suggests that designing teams should focus on both learning and game play simultaneously from the beginning of the design process [15]. Other important challenge is translating interest and potential into actual adoption and use [6]. Arnab et al. call attention to the fact that "despite the digital games' potential in terms of interactivity, immersion and engagement, more work is still required to understand how to better design, administrate and evaluate digital games across different learning contexts and targets" [6, p.392].

\subsection{Children Cognitive Development, Tablet Interaction and Game Instructions}

Previous studies on children computer interaction has shown that children are not as proficient as adults when interacting with tablet devices [4]. Children from different ages vary in their cognitive and motor skills and previous research have shown that younger children presented more difficulties to interact with tangible devices than children with a more advanced stage of cognitive development [3] [4]. As we previously discussed, our first serious game prototype focuses on children from 4 to 5 years old. According to Piaget's theory [19] [21], children from this age group are part of the Preoperational cognitive development stage ( 2 to 7 years old). In this stage children learn language skills, develop their motor skills and develop their memory and imagination.

Taking in account children's cognitive development previous researchers identified aspects that should be taken into consideration when designing tablet application for them, such as holding only one thing at memory at time [9] or manifesting centralization, focusing their attention in only one characteristic at time. Other studies identified several children-tablet interactions issues, which can be connected to children's continuously development of motor skills for instance: difficulties with multitouch [16] difficulties with drag and drop interaction [13] not recognized gestures [3] or misinterpreting touch actions [3] [16]. A common outcome from these studies is that children's interaction with tablet applications varies intensely from preschool to high-school age.

\subsection{Technology-Enhanced Learning for children without access to school}

Focusing in reducing the illiteracy previous researchers explored the use of mobile educational tools to promote learning in developing countries. The Pocket School project reviewed possible opportunities with mobile learning technology to counter the effects of the education inequality in Latin America [14]. Their results suggest that mobile learning technology has the potential to provide indigenous children with learning materials that could possibly teach them to read. The tablets were given to children in rural villages in Africa, and the results showed that the children, which took part in the study, encountered technology and literacy for the first time by using the tablet. Their results called attention to the highly collaborative aspect of children's tablet interaction in those communities and that children become passionate learners and also teachers of their community [9].

Another recent research describes a collaborative development of a digital, multi-language reading tool that facilitates translation of local stories by Kenyan community groups, led by a librarian, into a mother tongue [22]. The aim of the application is to break the chain of illiteracy, allowing parents with lowest levels of literacy explore stories with their children. A common outcome of these initiatives is that they demonstrated the value of applying mobile applications to teach children without access to schools. Unfortunately, each of them embrace a specific learning content, not providing a continuous education tool to be applied as a long term solution in the lack of regular schooling.

\section{SERIOUS GAME DESIGN}

As a result of the literature review and the collaborative multidisciplinary design process, we identified key challenges, and proposed solutions, to design a serious game for children out of schooling. As we will present in this section, the main challenge identified was the lack of teachers, and possibly also a lack of literate family members, to guide the learners throughout their education process. This issue was treated regarding three different aspects: motivation and engagement, identifying children's previous knowledge and knowledge transfer. Adding to it, other key design challenges were identified regarding learning and gaming balance, collaborative learning and Curriculum and children's cognitive development.

For the development of the prototype we centered on aspects related to motivation and engagement, knowledge transfer, learning vs. gaming balance, and children's cognitive development vs. tablet interaction. We summarize next the description of our design process, the challenges and proposed solutions regarding these four aspects.

\subsection{Design challenges and strategies}

Understanding that designing a serious game for children without access to school includes important aspects from different fields, a multidisciplinary research team worked in the design process. In total three low-fidelity prototypes were developed and discussed among the team. We present next a summary of the resulting design strategies and challenges, which were applied into the development of the prototype, that was later evaluated with children.

\subsubsection{Maintain Learner's Motivation and Engagement}

Taking into account that our target audience is children without access to school one of the main challenges for our serious game was to maintain children engagement with the application and their motivation to self-learning. From the literature review we understood the high potential of serious gaming for learning [6] 
Towards a mobile serious game environment for children selflearning

and value of RPG (Role Playing Games) to offer a motivating context that elicits engagement into language learning [18].

We applied a RPG game mechanics as a strategy to motivate and engage students into self-learning English and Mathematics. Aiming to do so, we combined RPG quest base structure [23] with the Quest based learning methodology [12] into the designing of an educational serious game.

\subsubsection{Quest Structure}

When playing the game learners will be presented with several quests in each level. For defining our quest representation we adopted the RPG's Linear Hub Pattern [23] adapting it to our educational proposes, Figure 1. In our serious game, a quest can serve as a learning purpose (teaching players school curriculum contents) or push forward the narrative. Each scenario corresponds to a specific learning competence and the different scenarios are connected by bridge quests. In this framework the learning activities are proposed to the players in the format of a RPG quest and will by often presented as mini-games. The quest starts with a simple objective and eventually narrow down to specific objectives of great significance. Learners receive a reward when completing these quests, which can be points or finding a magical artifact that will give him/her spells that may be used to solve future quests. Besides the learning quests, each scenario may also contain contextualizing quests, which are more connected to pushing forward the narrative or teaching players about the game design. The final learning activity from each competence corresponds to a debriefing activity (or a mini-game), where learners reflect in what they learned. In the end of each level the player will find an unlock quest. This quest allows players to move to the next level of the game.

\subsubsection{Pedagogical pattern}

In our serious game design we apply a pedagogical pattern that is dedicated to a learning that merges both learning and game into a same activity, placing the learner/player at the center of the educational program. This pattern bases on the educational Montessori approach combined with an instructional technique of modeling [17]. The first approach belongs to the epistemological principles in education and, more particularly, to a kind of constructivism relatively eclectic [17]. The second one belongs to the engineering of the learning systems, particularly, on-line learning systems. Both align themselves with constructivism as an instructional approach centered on the learner, more specifically, on his/her capacity to learn by him or herself.

Combining these two approaches, each activity of a learninggame process includes four steps: to explore, to manipulate, to operate, and to self-regulate. Each of these steps is presented to the learner/player in the format of a quest. The last step, to selfregulate, is a debriefing process and it is presented to the player as a bridge quest, and connects different learning scenarios. Following this pattern, the first two blocks of activities were designed. Each of them included four steps in an increasing level of complexity. These activities focused on the knowledge of the numbers from 1 to 5 and on introducing some words in English.

\section{PROTOTYPE EVALUATION}

Aiming to evaluate our design solutions, we developed a tablet serious game prototype and evaluated it with children. The goal of this study was to evaluate the key aspects of our design such as: children's enjoyment and motivation with the game; achievement of learning objectives; adequacy of the virtual learning environment and game elements; and aspects related to children's tablet interaction applied to serious games. Those outcomes will be applied as input to conduct our next research iteration and develop a further version of the serious game.

\subsection{Participants: profile and recruitment}

Since the serious game prototype was designed for children that would start the first class of schooling, we conducted the evaluation with preschoolers. 29 participants took part of the study, 13 parents and 16 children. Fifteen children were aged 4 years old, and one child already completed 5 years old, 8 girls and 8 boys. The participation on the study was voluntary and the participants were recruited in a multicultural french speaking Kindergarten in Montreal, Canada. . This location was chosen by its diversity, including canadian and immigrant families from different regions. First a pre-interview was conducted with the parents and later an evaluation activity was scheduled to be performed with the children during the Kindergarten hours.

\subsection{Serious game prototype}

To illustrate our design a prototype was developed for the Android device, more specifically for the Datawind's Ubislate tablet (Android version 4.2). Taking into consideration its technical specifications, our virtual learning environment is developed using 2D technology. The prototype was developed using the Unity3D game engine, which provides assets that facilitated our development process.

The prototype integrates the first four activities based on our Learning-Game Scenario pattern. The focus of these activities was at the learning the digits from 1 to 5 and introduce English vocabulary. The game storyline and instructions were presented with an audio in French, as most of the children did not domain the English language. The English vocabulary was introduced to children during the learning activities, which were presented as guest inside of the RPG game. The quests focused on searching for ingredients to cook a magical potion. The learner-player explores the vocabulary in order to memorize it and to match objects (fruit and digits) together. This matching between quantity of fruits and digits aims to favors the memorization.

The first two activities introduce the numbers and quantities from 1 to 5 . Each of them contains 5 exercises; after the children answer correctly, an animation counting the quantity of fruits or vegetables is triggered. Figure 2 shows part of these counting animations, related to activity 1 . In activity 3 , children have to 
connect the numbers with the correct quantity, as shown in Figure 3 . In the last activity, children are requested to choose the correct quantity of each required ingredient to do a magical potion, as shown in Figure 4. In the two first activities the interaction is based in touching the target objects and in activities 3 and 4 mainly in drag and drop.

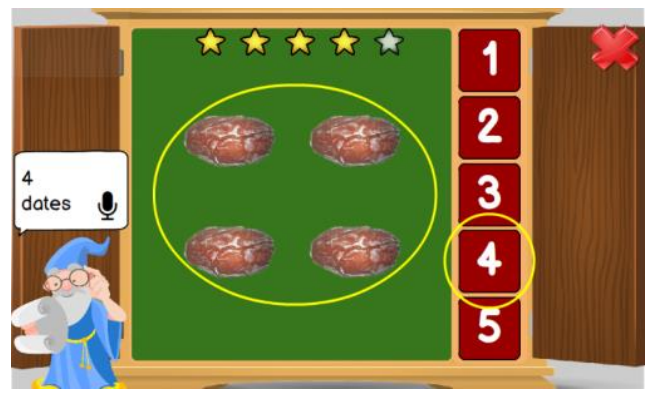

Figure 2 - Counting Activity

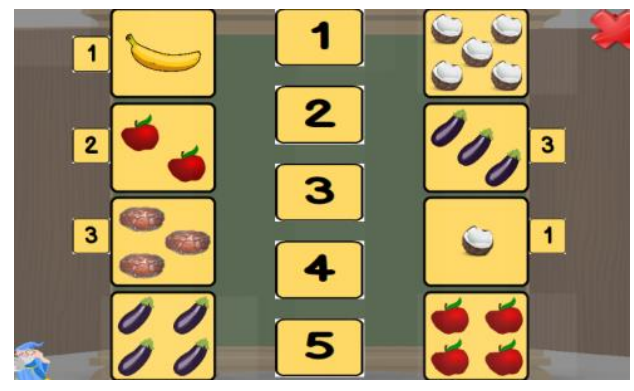

Figure 3-Matching numbers and quantities activity

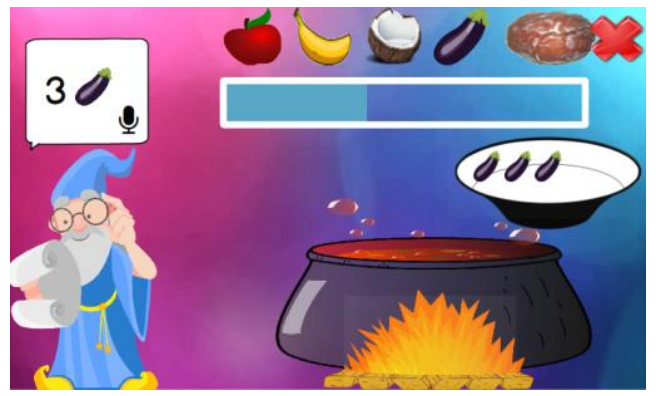

Figure 4- Selecting the right number of ingredients activity

When completing an activity correctly the children receive rewards in the format of magical objects. When finishing activity 1 and 3, children find a magical star and on completion of activity 2, the magical book. Moreover, when answering correctly an exercise, an encouraging audio and visual feedback are triggered.

\subsection{Data Collection}

The main objective of the evaluation is to value the effectiveness of the adopted design strategies. Our work especially focuses on children's motivation and enjoyment, the achievement of learning objectives and the learning / gaming balance, which are considered key aspects in serious game design [6] [7] [15].

Quantitative and qualitative research methods were applied in the data collection. Firstly, we conducted an interview with the children's parents. This interview aimed to identify children's previous experience with touch screen devices (phones and tablets), playing digital games, using educational tools and their previous knowledge with English. Later, we conducted the prototype evaluation with children, in which two researchers participated. One researcher focused on interacting with the child and running the activity. The other researcher, focused on the user observation and taking notes. An observation guide was developed to support the note taking process. Children played individually with the prototype and each section had approximately 30 minutes of duration. A video recording was used to record children's interaction with the tablet over there shoulder and to record there oral feedback that children provided through the activity. Prototype logs, for each playing section, were recorded to collect quantitative data regarding time, number of correct and wrong answers and outbound touches.

To evaluate the effectiveness of the learning tool, children were invited to answer a pre-test and a post-test. The tests were composed by two exercises in paper, inspired in the educational content presented in the prototype. Figure 5 shows a participant answering the pre test. To evaluate children enjoyment in relation to the game, and obtain extra feedback regarding the virtual learning environment and game elements, a short interview was conducted with the children after they play the game. This interview included 6 multiple-choice questions and one open question. For each of the multiple-choice questions children had to choose an answer demonstrated with smileys (1-5 scale).

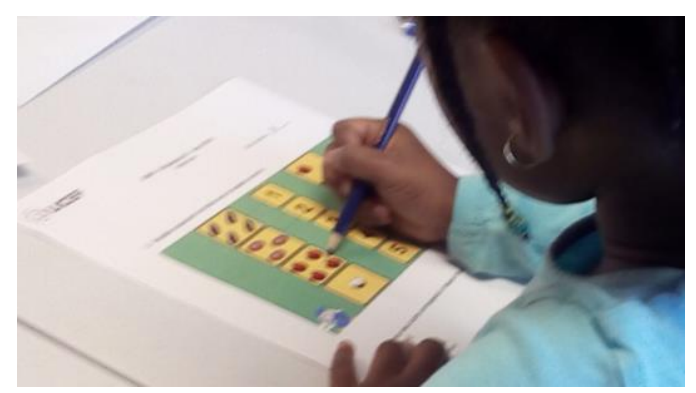

Figure 5 - Child answering the pre test.

\section{RESULTS AND DISCUSSION}

\subsection{Previous experience with touch devices, games, educational apps and English}

According to the interview with the parents $100 \%$ of the children had experience using mobile touch devices at home. $92.31 \%$ $(12 / 13)$ of them had previous experience with tablets and $69.23 \%$ (9/13) with smartphones. The parents affirmed that $38.46 \%(5 / 13)$ of the children used mobile touch devices every day, $38.46 \%$ $(5 / 13)$ three times per week and $23.08 \%$ (3/13) a few times per 
Towards a mobile serious game environment for children selflearning

month. They started to use touch devices with 2.33 years old (mean). In relation to their previous experience playing digital games, $38.46 \%(5 / 13)$ of the children play digital games every day, $30.77 \%$ (4/13) three times per week and $30.77 \%$ (4/13) a few times per month. According to the parents their frequency of use decline a bit in relation to educational apps with $30.77 \%$ (4/13) of the children using them every day, $23.08 \%(3 / 13)$ three times per week, $7.69 \%(1 / 13)$ once a week and $38.46 \%(5 / 13)$ a few times per month. Regarding previous contact with English language, $46.15 \%$ of the children utilized English at home or in other activities.

\subsection{Playtime and children's behavior}

The duration of the playtime was around 14 minutes, where the time for each activities varied from each child depending on interaction issues or difficulties with the learning content they had during the activity : Activity $1: 1.54 \mathrm{~min}$; Activity $2: 1.52 \mathrm{~min}$, Activity $3: 3.19 \mathrm{~min}$. and Activity $4: 5.00 \mathrm{~min}$. As the mean time shows, children took more time to complete activities 3 and 4 . This was mostly due the increased level of difficulty of these activities and difficulties children had with the instructions and interaction with the tablet.

By observing the facial and body expressions during the activity we classified that $75 \%(12 / 16)$ of the children were very focused and concentrated during the playing time. We also observed that $33.33 \%(4 / 12)$ of these children were smiling and/or celebrating in some occasions in the game (e.g.: when answering correctly an activity or receiving a game reward). Taking in consideration the rest of the children, on child $(6.25 \%)$ was smiling during most of the game play and 3 children $(18.75 \%)$ were mostly distracted during the activity, looking around in the room or towards the researchers. From these 3 children, 2 of them were smiling in some moments of the game play and one seemed a bit uncomfortable by not been able to easily proceed in the most difficult activities.

\subsection{Enjoyment with the game and game elements}

The results from the evaluation observation and children's interview show that the children enjoyed playing the game. During the evaluation all the children played the game until its completion, most of them were very concentrated during the activity and were willing to keep playing when the prototype finished. Similar outcomes from the observation were obtained from the interview with the children, in which they graded their enjoyment with the game as 4.44 (Mean, sd $=0.73$ ) and their intention to play the same game again as 4.25 (Mean, $\mathrm{sd}=0.77$ ), in a scale 1 (minimum) to 5 (maximum).

Other very important aspect connected to children's satisfaction with the game is their enjoyment with the game elements. Again both observation and children's feedback showed their engagement with the game elements. For instance, during the play sections we could notice some children smiling when, answering correctly one activity, receiving a game reward or choosing the avatar. Regarding children's evaluation about the game elements, in the interview they rated all elements above four. The Graphic 1 shows children's feedback in relation to the playing the game and different game elements, in a scale 1 (minimum) to 5 (maximum).

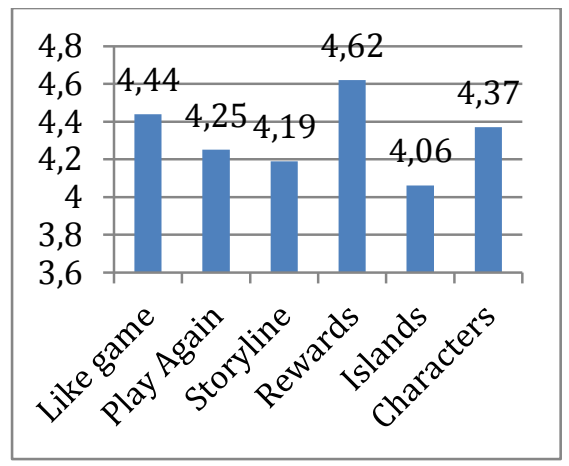

\section{Graphic 1 - Children's evaluation about serious game.}

\subsection{Complement to informal learning and individual self-learning}

From the pre tests we could observe that $44 \%$ (7/16) of the children already had good knowledge about the numbers from 1 to 5 , since they answered $100 \%$ correct on the numbers activity in the pre test. The situation was a bit different for recognizing the name of fruits and vegetables in English, in with only $12 \%(2 / 16)$ of the participants scored $100 \%$ in the pre test. Analyzing their results for the pre test for knowledge of numbers we obtained a mean of 5.5, sd $=2.61$ (maximum 8 ). Regarding the post text the mean was $5.94, \mathrm{sd}=2.46$ (maximum 8 ). If we exclude the children that already knew all the tested numbers (scoring $100 \%$ in the pre test) the results suggest that the remaining children learned on average 0.90 new numbers ( 4.34 mean post test -3.44 mean pre test). In relation to the words in English, the mean for the pre test was $2.56, \mathrm{sd}=1.36$ (maximum 5 ), and for the post test, the mean is 3.12 , $\mathrm{sd}=1.59$ (maximum 5).

This positive outcome suggests that the educational, and game design, approaches were appropriate for our participants. Even with this encouraging result we believe that further research, applying a more longitudinal evaluation approach, is necessary to fully understand how long should be the playing in order to children fully acquiring new competences, without the need of supplementary learning. From the experiments, we observed that the children that did not fully know the numbers in the pre test had difficulties with the numbers in the more complex game activities ( 3 and 4 ). Other interesting outcome from the evaluation was the value of the counting repetitions animations in activity 1 and 2. Many children followed these animations, either by moving their head or by talking loud repeating the numbers together with the game. This result encourages us to use similar strategy in other activities and also to consider other interaction strategies for the next version of the prototype. Into this direction, we aim to 
explore voice and gesture recognition as a possible interaction modality.

\subsection{Playing independently: Instructions, teaching strategy and interaction issues}

During the experiments we identified aspects that made difficult for children to play independently, where participants required help from the researcher to go further in the game. These aspects were related to needing help with the mini-games instructions, $100 \%(16 / 16)$ of the children, tablet interaction issues $62 \%$ (10/16) and requiring help because game teaching strategy was not acquired to their needs $44 \%(7 / 16)$.

In relation to understanding the game instructions, the difficulties increased as the activities got more complex. All children had difficulties with the instructions for activity 4 . This activity was more difficult in the pedagogical aspect but also required a more complex interaction to be completed. Besides providing and audio and virtual instructions about the learning content, an animation was presented in the beginning of the activity to teach the interaction steps. Besides the pedagogical complexity we observed two aspects that could be improved to facilitate this activity. The vast amounts of objects in the scene made difficult for children to notice the visual instructions, requiring the research to point to children where they should focus their attention. Secondly, the interaction animation in the beginning of the activity was not enough to guide children; a better solution would be to provide a guidance animation also when they were playing each step.

\section{CONCLUSIONS, LIMITATIONS AND FUTURE WORK}

The results of the evaluation show that the design of our educational tool as a RPG serious game was successful into motivating children with the learning activity. Children enjoyed playing with the learning game and gave positive feedback in relation to enjoying playing the game and being willing to play it again. This outcome suggests that such serious game approach could be successfully applied for engaging pre school children into educational activities. Regarding the adequacy of the pedagogical pattern, the results suggest that there was a small improvement on children's test grades, and as consequence in their knowledge, after playing the game. Even with this positive result we believe that further research (e.g. longitudinal evaluation) is necessary to fully understand how long should be the playing in order to children fully acquiring new competences, without the need of supplementary learning. s

As our current and future work, we aim to apply the results of this study in order to improve different aspects of the learning environment, such as the pedagogical pattern and game design, implement an integrated help and personalization systems. We want also to explore other tablet interaction strategies, such as voice and gesture recognition, manipulating physical objects and augmented reality, and evaluate its impact into children's tablet interaction and learning.

\section{ACKNOWLEDGMENTS}

We especially thank the Canadian funding agency MITACS to supporting this research, as well as the company Datawind R\&D Canada.

\section{REFERENCES}

[1] Clark C. Abt. 1970. Serious games: The art and science of games that simulate life. The Viking Press.

[2] J S K Achoka, Stephen O Odebero, Julius K Maiyo, and Ndiku J. Mualuko. 2007. Access to basic education in Kenya: inherent concerns. Educational Research and Reviews 2, 10: 275-284.

[3] Lisa Anthony, Quincy Brown, Jaye Nias, and Berthel Tate. 2013. Examining the need for visual feedback during gesture interaction on mobile touchscreen devices for kids. In Proceedings of the 12th International Conference on Interaction Design and Children - IDC '13, 157-164.

[4] Lisa Anthony, Quincy Brown, Jaye Nias, Berthel Tate, and Shreya Mohan. 2012. Interaction and recognition challenges in interpreting children's touch and gesture input on mobile devices. In Proceedings of the 2012 ACM international conference on Interactive tabletops and surfaces - ITS '12, 225.

[5] Kalene Ardt, et al. 2005. Report on primary education in bangladesh: Challenges and successes.

[6] Sylvester Arnab, et al. 2015. Mapping learning and game mechanics for serious games analysis. British Journal of Educational Technology 46, 2: 391-411.

[7] Francesco Bellotti, Bill Kapralos, Kiju Lee, Pablo Moreno-Ger, and Riccardo Berta. 2013. Assessment in and of Serious Games: An Overview. Advances in Human-Computer Interaction 2013: 1-11.

[8] R.M. Bottino, L. Ferlino, M. Ott, and M. Tavella. 2007. Developing strategic and reasoning abilities with computer games at primary school level. Computers \& Education 49, 4: 1272-1286.

[9] Angela Chang, Lidet Tilahun, and Cynthia Breazeal. 2014. Visualisations of Data from the Literacy Tablet Reading Project in Rural Ethiopia. In Proceedings of the Electronic Visualisation and the Arts (EVA), 142-149.

[10] Susan M. Ferreira, Sergio Sayago, and Josep Blat. 2016. Going Beyond Telecenters to Foster the Digital Inclusion of Older People in Brazil: Lessons Learned from a Rapid Ethnographical Study. Information Technology for Development. Retrieved July 20, 2016 from

[11] Jennifer Groff, Jody Clark-Midura, V. Elizabeth Owen, Louisa Rosenheck, and Michael Beall. 2015. Better learning in games: A balanced design lens for a new generation of learning games.

[12] Charles Christopher Haskell. 2012. Design Variables of Attraction in Questbased Learning. Boise State University. Retrieved March 11, 2016

[13] Lynne Humphries and Sharon McDonald. 2011. Emotion faces: the design and evaluation of a game for preschool children. In Proceedings of the 2011 annual conference extended abstracts on Human factors in computing systems (CHI EA'11), 1453.

[14] Paul Kim, Talia Miranda, and Claudia Olaciregui. 2008. Pocket School: Exploring mobile technology as a sustainable literacy education option for underserved indigenous children in Latin America. International Journal of Educational Development 28, 4: 435-445.

[15] Eric Klopfer, Scot Osterweil, and Katie Salen. 2009. Moving Learning Games Forward. Cambridge, MA. https://doi.org/10.1207/S15327825MCS0301_02

[16] Lorna McKnight and Daniel Fitton. 2010. Touch-screen Technology for Children: Giving the Right Instructions and Getting the Right Responses. In Proceedings of the 9th International Conference on Interaction Design and Children - IDC '10, 238.

[17] Elkind, D. 2003. Montessori and Constructivism. Montessori life, 15(1), 26.

[18] Mark Peterson. 2010. Massively multiplayer online role-playing games as arenas for second language learning. Computer Assisted Language Learning 23, 5: 429-439.

[19] Piaget and Jean. 1999. The Psychology Of Intelligence. Taylor \& Francis. Retrieved March 23, 2016

[20] Marc Prensky. 2001. Digital Game-based Learning. McGraw-Hill. Retrieved March 10, 2016

[21] Anik De Ribaupierre. 2001. Piaget's theory of child development. In International Encyclopedia of the Social and Behavioral Sciences. Oxford, $11434-11437$.

[22] Ariel Schwartz et al. 2015. Using a principal agent model to explain usercentered design challenges for mother tongue reading in Kenya. In Proceedings of the Seventh International Conference on Information and Communication Technologies and Development - ICTD '15, 1-8.

[23] Gillian Smith. 2009. RPG Design Patterns. Retrieved March 22, 2016

[24] UNESCO Institute for Statistics (UIS) and UNICEF. 2015. Fixing the broken promise of education for all: Findings from the Global initiative on Out-ofSchool Children. Montreal. 